\title{
RNA-Cholesterol Nanoparticles Function as Potent Immune Activators via TLR7 and TLR8
}

\author{
Hannah-Lena Obermann ${ }^{1 *}$, Ines I. Lederbogen ${ }^{1}$, Jenny Steele ${ }^{2}$, Jens Dorna ${ }^{1}$, \\ Leif Erik Sander ${ }^{2}$, Konrad Engelhardt ${ }^{3}$, Udo Bakowsky ${ }^{3}$, Andreas Kaufmann ${ }^{1}$ \\ and Stefan Bauer ${ }^{1 *}$ \\ 1 Institute for Immunology, Philipps-University Marburg, Marburg, Germany, ${ }^{2}$ Department of Infectious Diseases and \\ Pulmonary Medicine, Charité-Universitätsmedizin Berlin, Corporate Member of Freie Universität Berlin, Humboldt-Universität \\ zu Berlin, and Berlin Institute of Health, Berlin, Germany, ${ }^{3}$ Department of Pharmaceutics and Biopharmaceutics, Philipps- \\ University Marburg, Marburg, Germany
}

\section{OPEN ACCESS}

Edited by:

Ivan Zanoni,

Harvard Medical School, United States

Reviewed by:

David J. Dowling,

Boston Children's Hospital,

United States

Laura Pandolfi,

San Matteo Hospital Foundation

(IRCCS), Italy

${ }^{*}$ Correspondence:

Stefan Bauer

stefan.bauer@staff.uni-marburg.de

Hannah-Lena Obermann

hannah-lena.obermann@staff.uni-

marburg.de

Specialty section:

This article was submitted to

Molecular Innate Immunity,

a section of the journal

Frontiers in Immunology

Received: 26 January 2021

Accepted: 27 December 2021

Published: 21 January 2022

Citation:

Obermann H-L, Lederbogen II,

Steele J, Dorna J, Sander LE,

Engelhardt K, Bakowsky U,

Kaufmann A and Bauer S (2022)

RNA-Cholesterol Nanoparticles

Function as Potent Immune

Activators via TLR7 and TLR8.

Front. Immunol. 12:658895. doi: 10.3389/fimmu.2021.658895
The innate immune system senses viral and bacterial ribonucleic acid (RNA) via pattern recognition receptors (PRR) leading to subsequent activation of the immune system. One group of RNA sensors is formed by endosomal/lysosomal Toll-like receptors (TLR) such as TLR7 and TLR8. During viral or bacterial infection, immunostimulatory RNA is part of the pathogen reaching the endosomal/lysosomal compartment after cellular uptake. Synthetic single-stranded or double-stranded oligoribonucleotides (ORN) can mimic RNA from pathogens and are widely used as activating ligands for TLR7 and TLR8. However, one limitation in the use of synthetic ORN driven immune stimulation is the need for transfection reagents for RNA delivery into cells. Here we demonstrate that the conjugation of cholesterol to a double-stranded version of immunostimulatory RNA40 strongly enhanced RNA uptake into monocytes and plasmacytoid dendritic cells when compared to naked RNA. Cholesterol-conjugated RNA (RNA-chol) formed nanoparticles that were superior to RNA-liposomes complexes in regard to induction of type I interferon from human and murine plasmacytoid dendritic cells as well as proinflammatory cytokine production (e.g. TNF- $\alpha$, IL12p70 or IL-6) in human monocytes. Furthermore, the RNA40chol induced cytokines in human monocyte cultures supported $T_{H 1}$ and $T_{F H}$ cell differentiation underscoring a strong adjuvant function of RNA-chol nanoparticles for adaptive immune responses. In summary, cholesterol-conjugated immunostimulatory RNA forms nanoparticles and functions as a potent immune adjuvant in human and murine immune cells. It further simplifies the use of immunostimulatory RNA by avoiding the need for liposomal transfection reagents.

Keywords: TLR7/8 ligand, RNA-cholesterol, nanoparticle, immunostimulation, dsRNA

\section{INTRODUCTION}

The synthetic immunostimulatory toll-like receptor 7- (TLR7) and TLR8-ligand (TLR8L) ribonucleic acid 40 (RNA40), which is a single-stranded (ss) GU-rich sequence from the U5 region of HIV-1 RNA, and its potential to act as adjuvant or for immunotherapy has already been described (1). In addition, double-stranded (ds) short interfering (si) RNA is a potent inducer of interferon- $\alpha$ (IFN- $\alpha$ ) secretion in 
murine plasmacytoid dendritic cells (pDC) through activation of TLR7 (2). TLR7 and TLR8 belong to a subgroup of pattern recognition receptors (PRR) that is located within the endosomal/ lysosomal compartment (3) of immune cells and sense RNA degradation products: TLR7 recognizes guanosine and uridine moieties in ssRNA (4) and TLR8 detects uridine and a short oligonucleotide created by RNase T2 and/or RNase 2 (5-7). Since unmodified nucleic acids hardly interact with cell membranes and are highly prone to degradation by DNases or RNases, cellular uptake of synthetic TLRL has to be facilitated by complexing the nucleic acids to transfection reagents like DOTAP. A major drawback of this method is the additional step of complex formation, which has to be performed prior to adding the TLRL to target cells. For this reason, developing TLRL that can be directly added to and activate target cells is highly desirable. Among other molecules, cholesterol-conjugation has been shown to: i) improve nuclease resistance of oligodeoxynucleotides (ODN) (8), ii) mediate cellular uptake of siRNA across plasma membranes $(9,10)$ and iii) induce an immune response by ssRNA with phosphorothioate linkage (11). In addition, cholesterol-conjugation of nonmethylated CpG-DNA leads to formation of nanoparticles by self-assembly (12). Conjugation of cholesterol to siRNA and antisense oligodeoxynucleotides and their cellular uptake as well as in vivo distribution has been extensively investigated $(9,10,13-$ 16). In contrast, cholesterol-conjugation to immunostimulatory phosphodiester RNA (e.g. dsRNA40) in order to activate the innate immune system has not been studied in detail. Due to the ability of macrophages and dendritic cells to incorporate nano- and microparticles via phagocytosis or endocytosis, these particles possess the potential to act as drug or adjuvant delivery system. In this study, we investigated the potential of a cholesterolconjugated dsRNA [based on the sequence of the TLR7/TLR8 ligand RNA40 (1)] to form nano- or microparticles and activate TLR7 and TLR8. Therefore, we analyzed cellular uptake and the immunostimulatory potential of cholesterol-conjugated dsRNA40 by murine Flt3L-DC, human $\mathrm{pDC}$ and human monocytes and analyzed the contribution of RN40-chol-induced immunostimulation to $\mathrm{T}$ cell differentiation.

\section{MATERIALS AND METHODS}

\section{Ethics Statement}

The local ethics committees of Justus-Liebig-University Giessen and Philipps-University Marburg approved the use of human blood samples for this study. For experiments with murine immune cells, mice were sacrificed, and tissue/organs removed. These experiments were performed in accordance with the National German welfare law $\$ 4$ (3) TierSchG and $\$ 2$ and Annex 2 (TierSchVerV) of the National Order for the use of animals in research and do not need the approval by a local ethics committee. According to the regulations, the number of mice used was reported to the animal welfare officer of the Philipps-University Marburg.

\section{Reagents}

ssRNA40sense (5'-GCCCGUCUGUUGUGUGACUC), GU-rich sequence from the U5 region of HIV-1 RNA, nt 108-127 (1); ss RNA40 - chol (5'- GCCCGUCUGUUGUGUGACUCcholesteryl), ssRNA40antisense (5'-GAGUCACACAACAGACG GGC), ssRNA40anti-2'O-methyl (5'-GaGuCaCaCaAcAgAc $\mathrm{GgGc}$ ) where small letters indicate 2'O-methylated positions and ssRNA40antisense-Alexa488 (ssRNA40antisense (5'GAGUCACACAACAGACGGGC-Alexa488) were synthesized by IBA (Göttingen, Germany) or BioSpring GmbH (Frankfurt, Germany). The sequence of CpG-ODN 2216 (5' GsGsGGGACGATCGTCsGsGsGsGsGsG), where 's' depicts a phosphorothioate linkage, was synthesized by TIB MOLBIOL (Berlin, Germany). Resiquimod (R848), TL8-506 were purchased from InvivoGen (San Diego, USA), LPS from Sigma Aldrich (Steinheim, Germany) and DOTAP was purchased from Carl Roth (Karlsruhe, Germany) or Roche (Basel, Switzerland). Flt3-ligand (Flt3L) was prepared from an Flt3L-secreting cell line (H. Hochrein, Bavarian Nordic GmbH, Martinsried, Germany). Antibodies were purchased from BD Biosciences (rat anti-mouse B220-FITC), eBioscience (rat anti-mouse CD11c-APC), Miltenyi Biotec (mouse anti-human BDCA-2-FITC, mouse anti-human CD14-PE) and Biolegend (mouse anti-human CD3, mouse antihuman CD28, mouse anti-human CD4 and mouse anti-human IFN- $\gamma$ ).

\section{Annealing of dsRNA}

For the generation of dsRNA40, ssRNA40sense and ssRNA40antisense were annealed. dsRNA40-chol was created by annealing ssRNA40-chol and ssRNA40antisense, whereas dsRNA40-2'OM was generated by annealing ssRNA40sense and ssRNA40anti-2'O-methyl. In brief, equal molar amounts of complementary single-stranded RNA molecules were combined in annealing buffer (20 mM HEPES, $150 \mathrm{mM} \mathrm{NaCl}$ ) and the solution was heated to $95^{\circ} \mathrm{C}$ for ten minutes and slowly cooled down to room temperature. Formation of doublestranded RNA (final concentration of $20 \mu \mathrm{M}$ ) was verified by $15 \%$ non-denaturing polyacrylamide gel-electrophoresis.

\section{Mice}

TLR7-deficient mice were established as described and have been backcrossed to C57BL/6 mice for at least 12 generations (17). C57BL/ 6 wild type mice were bred in the animal facility of the Philipps-University Marburg. Mice were kept under specific pathogen-free conditions in the animal facility of the PhilippsUniversity Marburg at the biomedical research center.

\section{Cells}

To differentiate Flt3L-induced mixed cultures of murine plasmacytoid and myeloid dendritic cells, bone marrow cells were seeded at $1.5 \times 10^{6}$ cells $/ \mathrm{ml}$ in Opti-MEM (Thermo Fisher Scientific, Waltham, USA) supplemented with $0.05 \mathrm{mM} \beta$ mercaptoethanol (Thermo Fisher Scientific, Waltham, USA), $100 \mathrm{U} / \mathrm{ml}$ penicillin (PAA, Cölbe, Germany), $100 \mu \mathrm{g} / \mathrm{ml}$ streptomycin (PAA, Cölbe, Germany), 1\% FCS (Biochrom AG, Berlin, Germany) and cultured with the Flt3L containing supernatant in a 1:250 dilution for seven days. Bone marrow derived macrophages (BMDM) were generated using $20 \mathrm{ng} / \mathrm{ml}$ murine M-CSF (Peprotech, Rocky Hill, USA) with $5 \times 10^{6}$ cells/ $10 \mathrm{ml}$ in RPMI1640 supplemented with $0.05 \mathrm{mM} \beta$ - 
mercaptoethanol (Thermo Fisher Scientific, Waltham, USA), $100 \mathrm{U} / \mathrm{ml}$ penicillin (PAA, Cölbe, Germany), $100 \mu \mathrm{g} / \mathrm{ml}$ streptomycin (PAA, Cölbe, Germany) and cultured for 5 days. Three days after isolation additional $20 \mathrm{ng} / \mathrm{ml} \mathrm{M-CSF}$ was added. Human PBMC were isolated by Ficoll density gradient centrifugation using lymphocyte separation medium LSM1077 (PAA, Cölbe, Germany) according to the manufacturer's recommendation. Untouched human $\mathrm{pDC}$ and untouched human monocytes were isolated with the plasmacytoid dendritic cell isolation kit II or the monocyte isolation kit II (Miltenyi Biotec, Bergisch-Gladbach, Germany) from PBMC according to the manufacturer's recommendation. Cells were routinely analyzed by flow cytometry (FACSCalibur, BD Biosciences, San Jose, USA) to assess purity.

\section{Cell Stimulation}

Murine Flt3L-induced DC and human monocytes were seeded at $2 \times 10^{5}$ cells/well and human pDC were seeded at $0.15 \times 10^{5}$ cells/ well. Murine Flt3L-induced DC were cultured in Opti-MEM (Thermo Fisher Scientific, Waltham, USA) supplemented with $0.05 \mathrm{mM} \beta$-mercaptoethanol (Thermo Fisher Scientific, Waltham, USA), $100 \mathrm{U} / \mathrm{ml}$ penicillin (PAA, Cölbe, Germany), $100 \mu \mathrm{g} / \mathrm{ml}$ streptomycin (PAA, Cölbe, Germany), 1\% FCS (Biochrom AG, Berlin, Germany). Human monocytes and pDC were cultured in RPMI 1640 (PAA, Cölbe, Germany) supplemented with $2 \mathrm{mM}$ L-glutamine (PAA, Cölbe, Germany), $100 \mathrm{U} / \mathrm{ml}$ penicillin (PAA, Cölbe, Germany), 100 $\mu \mathrm{g} / \mathrm{ml}$ streptomycin (PAA, Cölbe, Germany), 1x non-essential amino acids (PAA, Cölbe, Germany), $1 \mathrm{mM}$ sodium pyruvate solution (PAA, Cölbe, Germany). Human $\mathrm{pDC}$ and monocytes were stimulated without serum.

Cells were stimulated with ssRNA40 or dsRNA40 complexed to DOTAP as well as dsRNA40-chol, and dsRNA40-chol-2'O-methyl at concentrations as indicated. After 20 hours supernatants were harvested and analyzed for cytokine secretion. Stimuli diluted in Opti-MEM in a total of $25 \mu \mathrm{l}$ were mixed with $25 \mu \mathrm{l}$ DOTAPsolution $(2.5 \mu \mathrm{l}$ DOTAP and $22.5 \mu \mathrm{l}$ Opti-MEM) and incubated for ten minutes at room temperature before an equal volume of medium was added. For stimulation without complexing stimuli to DOTAP, DOTAP-solution was replaced by Opti-MEM. Cells stimulated with medium, DOTAP, CpG2216 $(1 \mu \mathrm{M})$, LPS $(1 \mu \mathrm{g} / \mathrm{ml})$ in combination with $\mathrm{R} 848(2.5 \mu \mathrm{g} / \mathrm{ml})$ served as negative and positive controls, respectively. Cells within $100 \mu \mathrm{l}$ volume were incubated with $100 \mu \mathrm{l}$ of stimuli in 96-well microplates (Greiner Bio-one, Gremsmünster, Austria). Cytokines were analyzed by ELISA with reagents from PBL (Piscataway, USA) for murine IFN- $\alpha$ or Peprotech (Rocky Hill, USA) for murine TNF- $\alpha$, murine CXCL10, murine and human IFN- $\beta$ or R\&D Biosystems (Minneapolis, USA) for murine IL- 6 and human IL- $1 \beta$ or BD Biosciences (San Jose, USA) for human IL- 6 and TNF- $\alpha$ or Bender MedSystems (Wien, Austria) and BD Biosciences (San Jose, USA) for human IFN- $\alpha$ and eBioscience (San Diego, USA) for human IL-12p70.

\section{In Vitro T Cell Priming}

Human PBMC were isolated as described above. $\mathrm{CD}^{+} \mathrm{T}$ cells were isolated from PBMC with the MagniSort Human CD4 T cell enrichment kit (eBioscience, San Diego, USA) according to the manufacturer's recommendation. $\mathrm{CD}^{+} \mathrm{T}$ cells were seeded at $0.5 \times 10^{5}$ cells/well within $100 \mu \mathrm{l}$ RPMI 1640 (GIBCO/Invitrogen, Carlsbad, CA, USA) supplemented with $1 \%$ non-essential amino acids (Sigma, München, Germany), 1\% HEPES (Sigma, München, Germany), 1\% GlutaMAX supplement (Thermo Fisher Scientific, Waltham, USA), 1\% penicillin/streptomycin (Sigma, München, Germany) and 10\% heat-inactivated autologous plasma) into antiCD3 $\left(4 \mu \mathrm{g} / \mathrm{ml}\right.$ ) coated Corning ${ }^{\circledR}$ 96-well plates (Corning, New York, USA). $100 \mu \mathrm{l}$ of conditioned monocyte supernatant (monocytes were stimulated as described above) and $1 \mu \mathrm{g} / \mathrm{ml}$ anti-CD28 were added to the $\mathrm{CD} 4^{+} \mathrm{T}$ cells. After five days of incubation, cells were analyzed for intracellular IFN- $\gamma$, IL-21 and Bcl-6. Therefore, PMA (Sigma, München, Germany) and ionomycin (Sigma, München, Germany) were added at $50 \mathrm{ng} /$ $\mathrm{ml}$ and $1 \mu \mathrm{g} / \mathrm{ml}$ to $\mathrm{CD}^{+}{ }^{+} \mathrm{T}$ cells. Cells were incubated for 2.5 hours before 1x brefeldin A (eBioscience, San Diego, USA) and $1 \mathrm{x}$ monensin (Biolegend, San Diego, USA) were added and cells were incubated for additional 2.5 hours. Cells were harvested and stained for CD4 and intracellular IFN- $\gamma$, IL-21 and Bcl-6. For the staining of IFN- $\gamma$ and IL-21, cells were fixed and permeabilized with cytofix/cytoperm fixation solution and perm/wash buffer as described in the operation manual of the manufacturer $\mathrm{BD}$ Biosciences. For the staining of Bcl-6, cells were fixed and permeabilized with the Foxp3/transcription factor staining buffer set (eBioscience, San Diego, USA) as described in the operation manual. Cells were analyzed with a FACS CANTO II and the FACS Diva software, which was available in the flow cytometry lab of the BCRT, Charite Universitätsklinikum Berlin.

\section{Photon Correlation Spectroscopy (PCS) and Laser Doppler Velocimetry (LDA)}

The hydrodynamic diameter and zeta potential of the dsRNA-chol particles were measured by PCS and LDV respectively using Zetasizer Nano ZS (Malvern Instruments, Herrenberg, Germany) equipped with $10 \mathrm{~mW} \mathrm{HeNe}$ laser at a wavelength of $633 \mathrm{~nm}$ at $25^{\circ} \mathrm{C}$. A viscosity of $0.88 \mathrm{mPa} \times \mathrm{s}$ and a refractive index of 1.33 of water at $25^{\circ} \mathrm{C}$ were assumed for data interpretation. Laser attenuation and measurement position were adjusted automatically by the instrument. The zeta potential was measured via electrophoretic mobility with laser Doppler velocimetry (LDA). The average values of the size intensity peak, the size volume peak, $\mathrm{z}$-average $(\mathrm{z}$-AvE) and zeta potential were calculated with data of three independent experiments \pm standard deviation. Each sample was measured three times with at least 10 sub runs.

\section{Atomic Force Microscopy (AFM) Measurements}

AFM was performed using a NanoWizard ${ }^{\circledR} 3$ atomic force microscope (JPK Instruments, Berlin, Germany). Silicon cantilevers (HQ : NSC14/AL_BS, MikroMasch Europe, Wetzlar, Germany) were used to measure the dsRNA40-chol particles. Measurements were performed in intermittent contact mode to avoid damage of the sample (18). Images were obtained by displaying the amplitude signal of the cantilever in the trace direction and the measured height mode in retrace direction. For diameter measurements the height mode signal in trace direction 
are used. The images of the particles were presented in amplitude mode.

\section{Confocal Laser Scanning Microscopy}

Human pDC were incubated with $0.5 \mu \mathrm{M}$ of Alexa488-labeled dsRNA40-chol, dsRNA40 or dsRNA40 complexed to DOTAP. Mixtures were prepared as described for cell stimulations. After 1.5 hours of incubation, cells were extensively washed to remove any free nucleic acids. Cells were then fixed with $2 \%$ paraformaldehyde (Carl Roth, Karlsruhe, Germany). Nuclei were stained with $0.1 \mu \mathrm{g} / \mathrm{ml}$ of DAPI (Merck, Darmstadt, Germany) and pictures were taken with a TCS SP5 confocal laserscanning microscope (original magnification x63) (Leica Microsystems, Wetzlar, Germany). Images were processed and plot profiles were generated with Fiji (ImageJ) software.

\section{Flow Cytometry}

Primary cells were incubated with $0.5 \mu \mathrm{M}$ of Alexa488-labeled dsRNA40-chol, dsRNA40 or dsRNA40 complexed to DOTAP. Mixtures were prepared as described for cell stimulations and confocal laser scanning microscopy. Cells were analyzed with a FACSCalibur (BD Biosciences, San Jose, USA).

\section{Statistical Analysis}

Statistical significance was analyzed using unpaired t-test or oneway ANOVA followed by post-hoc tests as indicated to correct for multiple comparisons. Data calculations were performed using Graph Pad Prism 8.4.1 software. Bars show mean + SEM of biological replicates. The values of $\mathrm{p}<0.05$ were considered to be statistically significant $\left({ }^{*} \mathrm{p}<0.05,{ }^{* *} \mathrm{p}<0.01,{ }^{* *} \mathrm{p}<0.001\right.$, $\left.{ }^{* * * *} \mathrm{p}<0.0001\right)$.

\section{RESULTS}

\section{Cholesterol-Conjugated dsRNA40 Induces Potent Immunostimulation in Plasmacytoid Dendritic Cells and Human Monocytes in a TLR7- and TLR8-Dependent Manner}

In this study, we addressed the immunostimulatory potential of cholesterol-conjugated RNA40. The RNA-sequence used in our study is based on the TLR7 and TLR8 ligand ssRNA40 (1). At first, we analyzed, whether ssRNA40-chol (ssRNA40 with cholesterol-conjugation at the 3'-end) could induce cytokine production in murine Flt3L-DC. In contrast to DOTAPmediated transfection of ssRNA40, ssRNA40-chol did not significantly induce secretion of interferon- $\alpha$ (IFN- $\alpha$ ), interleukin-6 (IL-6), tumor necrosis factor- $\alpha$ (TNF- $\alpha$ ) or CXCL10 in comparison to DOTAP-mediated transfection of ssRNA40 (Figure 1A). Since ssRNA40 could rapidly be digested by cellular RNases, we analyzed the immunostimulatory potential of cholesterol-conjugated dsRNA40, which should be more resistant against degradation by $\mathrm{RNases}$. Indeed, dsRNA40-chol induced significantly higher IFN- $\alpha$ and TNF- $\alpha$ levels compared to dsRNA40 complexed to DOTAP (Figure 1B). The cytokines IL- 6 and CXCL10 were also induced by dsRNA40- chol (Figure 1B). In addition, we wanted to exclude the possibility of cholesterol-induced cytokine release. Because alternating 2'O-ribose-methylation (2'OM) has been described to render RNA non-stimulatory (19-25) due to the resistance to RNase digestion (7), we stimulated Flt3L-DC with dsRNA40chol-2'OM to silence dsRNA40-mediated immune activation. Accordingly, we could not detect any cytokine-induction arguing against stimulatory activity of cholesterol (Figure 1B). Furthermore, dsRNA40-chol-induced cytokine production was strictly dependent on TLR7 since cytokine response was blunted in TLR7-deficient cells (Figure 1B). For all experiments on the immunostimulatory potential of dsRNA40-chol, ssRNA40-chol (ssRNA40 with cholesterol-conjugation at the 3'-end) and ssRNA40 antisense were annealed at an equal molar ratio and double-strand formation was analyzed by non-denaturing polyacrylamide gel electrophoresis (PAGE) (Figure 1C). dsRNA40-chol showed effective annealing and ssRNA40-chol and dsRNA40-chol migrated slower than corresponding unconjugated RNA. Sequences of ssRNA40 and dsRNA40 are also shown in Figure 1C.

Due to the fact that dsRNA40-chol leads to TLR7-mediated immunostimulation in the murine system, we purified human pDC and analyzed IFN- $\alpha$ secretion. Indeed, dsRNA40-chol induced IFN- $\alpha$ in human $\mathrm{pDC}$ and IFN- $\alpha$ levels were increased with lower concentrations of dsRNA40-chol. In contrast, IFN- $\alpha$ levels induced by dsRNA40 complexed to DOTAP were generally lower and decreased with lower concentrations. This observation supports the conclusion that dsRNA40-chol is superior to dsRNA40 complexed to DOTAP at a concentration of $0.1 \mu \mathrm{M}$ dsRNA40 (Figure 1D). Again, dsRNA40-chol with alternating 2'O-ribose-methylation at the antisense strand showed no immunostimulatory activity (Figure 1D).

We further extended the analysis of cholesterol-modified RNA40 mediated immune stimulation to murine macrophages and human monocytes (Figure 2). The response of murine bone marrow derived macrophages (BMDM) to ssRNA40 was detectable for IFN- $\beta$ and IL- 6 in a DOTAP- dependent manner, whereas only cholesterol-coupled ssRNA40 induced TNF- $\alpha$ (Figure 2A). Unexpectedly, dsRNA40-chol did not induce cytokines in BMDM, however DOTAP-complexed dsRNA40 induced IFN- $\beta$, but no IL-6 or TNF- $\alpha$ (Figure 2A). In contrast, purified human monocytes responded strongly to dsRNA40-chol with IL- 6 and TNF- $\alpha$ production, however type I interferon was not induced (Figure 2B). Strikingly, dsRNA40chol induced cytokine production was strictly TLR8-dependent since the TLR8 inhibitor Cu-CPT9a blunted cytokine production (Figure 2B). DOTAP-transfected dsRNA40 also induced IL-6 and TNF- $\alpha$ in a TLR8-dependent manner although at lower levels when compared to dsRNA40-chol. The specificity of the TLR8 inhibitor was verified by demonstrating that cytokine production by stimulation with LPS, a TLR4 ligand, was not influenced by $\mathrm{Cu}-\mathrm{CPT} 9 \mathrm{a}$. Of note, IFN- $\alpha$ and IFN- $\beta$ were only induced by DOTAP-transfected dsRNA40 in a TLR8independent manner. A response of contaminating pDCs can be ruled out, since the TLR9 ligand CpG2216, a strong IFN- $\alpha$ inducer in pDCs, did not induce any type I interferon in purified 

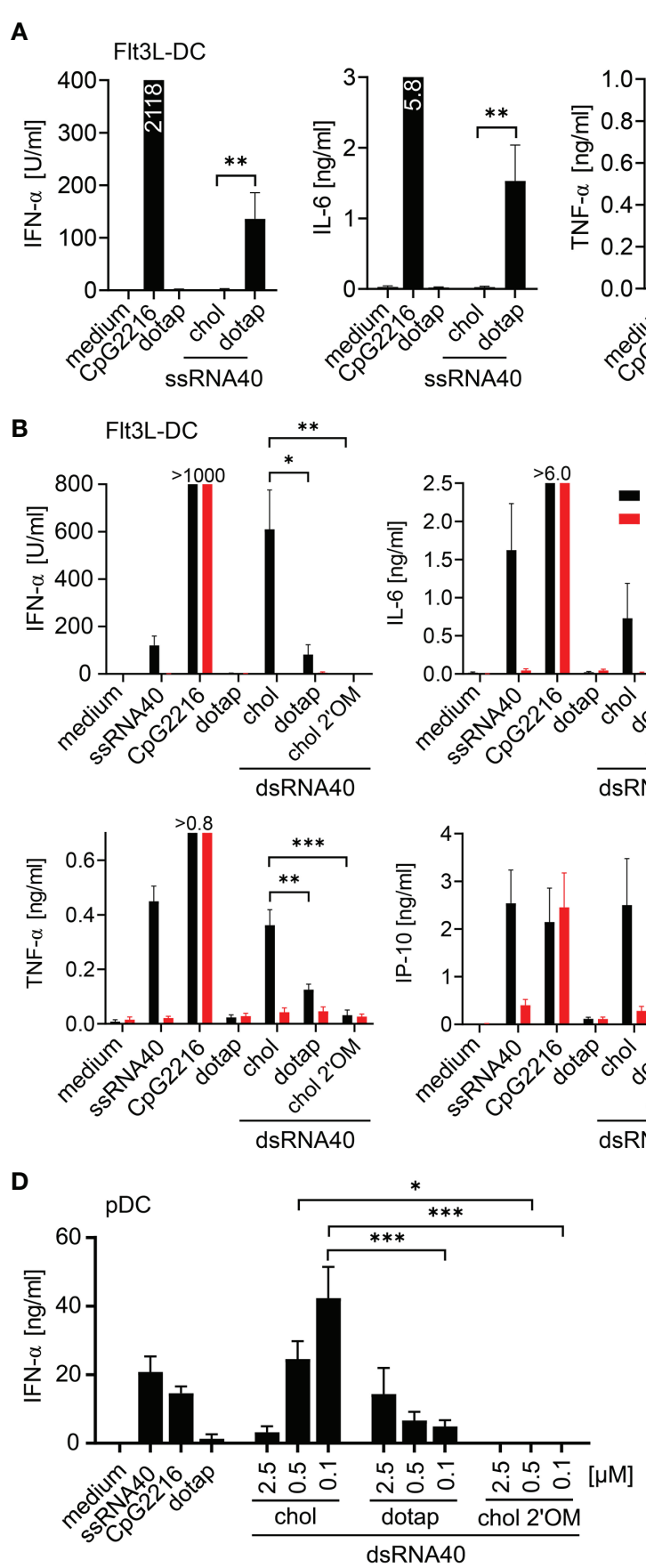
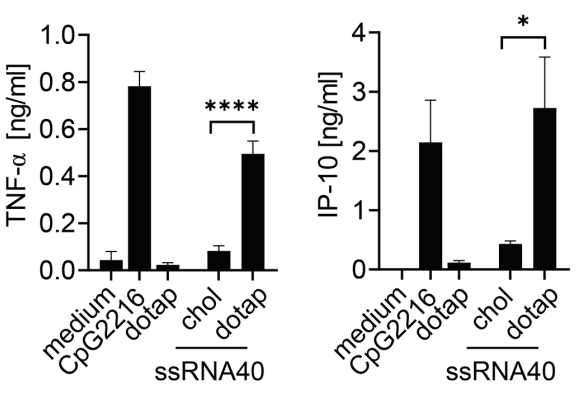

C
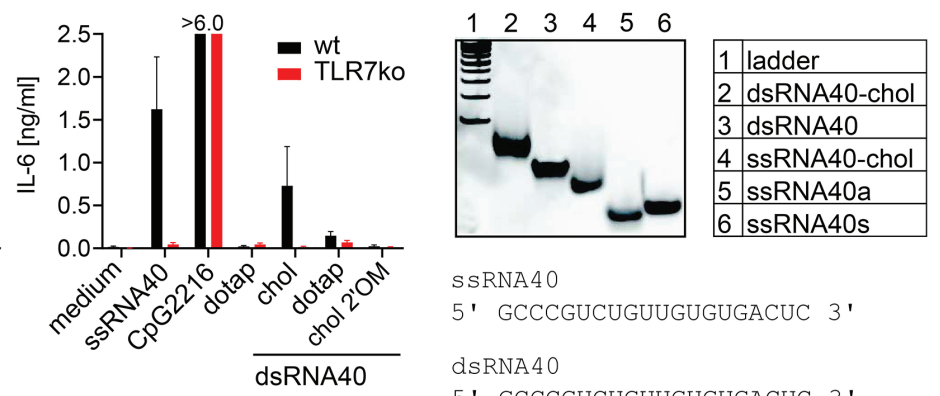

SSRNA 40

5' GCCCGUCUguUgugugAcuC 3'

dSRNA 40

5' GCCCGUCUGUUGUGUGACUC 3 '

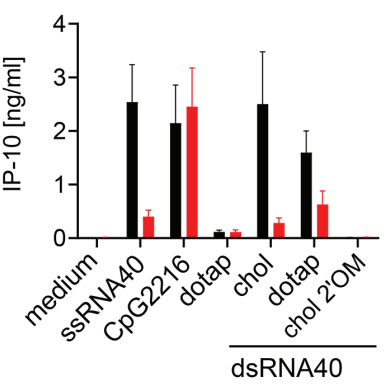

3. CGGGCAGACAACACACUGAG 5 '

FIGURE 1 | Cholesterol-conjugated dsRNA40 induces a potent immunostimulation. (A) Wt Flt3L-DC were stimulated with 0.5 MM ssRNA40-chol or ssRNA40 complex to DOTAP. Medium, CpG2216 (1 $\mathrm{MM})$ and DOTAP served as controls. Supernatants were taken after $20 \mathrm{~h}$ of incubation and cytokine production was analyzed by ELISA. Data were calculated from at least five individual experiments. Bars indicate mean $+\mathrm{SEM}$. ${ }^{\star} \mathrm{p}<0.05$, ${ }^{\star \star} \mathrm{p}<0.01$, ${ }^{\star \star \star \star} \mathrm{p}<0.0001$; unpaired t-test. (B) Wt and TLR7-deficient Flt3L-DC were incubated with $0.5 \mu \mathrm{M}$ dsRNA40-chol, dsRNA40 complexed to DOTAP and dsRNA40-chol-2'OM. Medium ssRNA40 $(0.75 \mu \mathrm{M})$ complexed to DOTAP, CpG2216 (1 $\mu \mathrm{M})$ and DOTAP served as controls. Cytokine production was analyzed by ELISA after $20 \mathrm{~h}$ of incubation. CpG induced $>1000 \mathrm{U} / \mathrm{ml} \mathrm{IFN-} \alpha$ in wt and TLR7-deficient cells, $6.3 \mathrm{ng} / \mathrm{ml} \mathrm{IL}-6$ in wt, $6.9 \mathrm{ng} / \mathrm{ml} \mathrm{IL-6} \mathrm{in} \mathrm{TLR7-deficient} \mathrm{cells} \mathrm{and} 0.8 \mathrm{ng} / \mathrm{ml}$ TNF- $\alpha$ in wt and $1.0 \mathrm{ng} / \mathrm{ml} \mathrm{TNF}-$ $\alpha$ in TLR7-deficient cells. Data were calculated from five to seven individual experiments. Bars indicate mean $+\mathrm{SEM}$. ${ }^{\star} \mathrm{p}<0.05,{ }^{\star \star} \mathrm{p}<0.01,{ }^{\star \star \star} \mathrm{p}<0.001 ;$ one-way ANOVA with Sidak's post-hoc test. (C) Polyacrylamide gel electrophoresis of annealed dsRNA40-chol and corresponding sequences. Nucleic acids were stained with SybrGold. One representative gel out of three is shown. (D) Purified human pDC were stimulated with dsRNA40-chol, dsRNA40 complexed to DOTAP and dsRNA40-chol-2'OM at $2.5 \mu \mathrm{M}, 0.5 \mu \mathrm{M}$ and $0.1 \mu \mathrm{M}$. Medium, ssRNA40 (0.75 $\mu \mathrm{M}), \mathrm{CpG} 2216(1 \mu \mathrm{M})$ and DOTAP served as controls. Supernatants were harvested after $20 \mathrm{~h}$ of incubation and IFN- $\alpha$ was analyzed by ELISA. Data were calculated from three independent experiments. Bars indicate mean + SEM. ${ }^{\star} p<0.05$, ${ }^{\star \star \star} \mathrm{p}<0.001$; one-way ANOVA with Sidak's post-hoc test. 
A
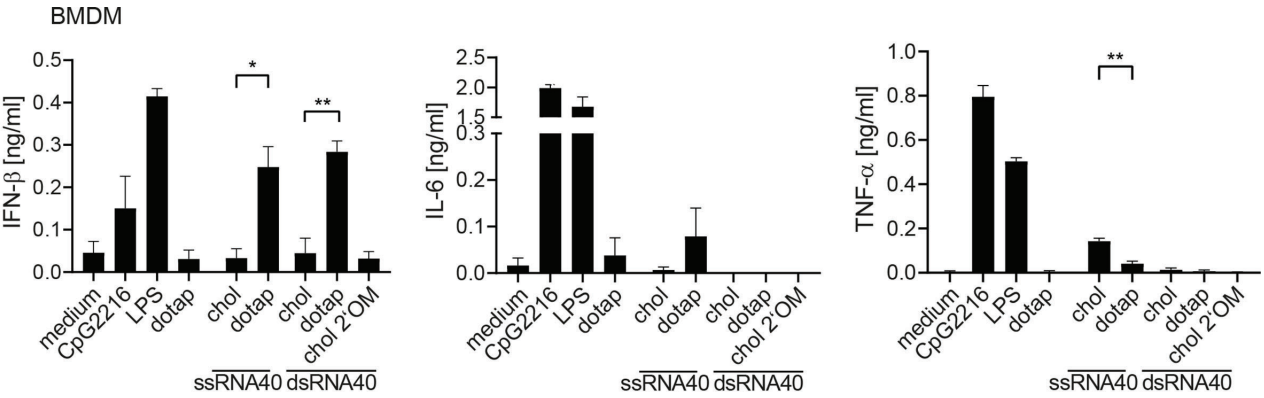

B Human Monocytes
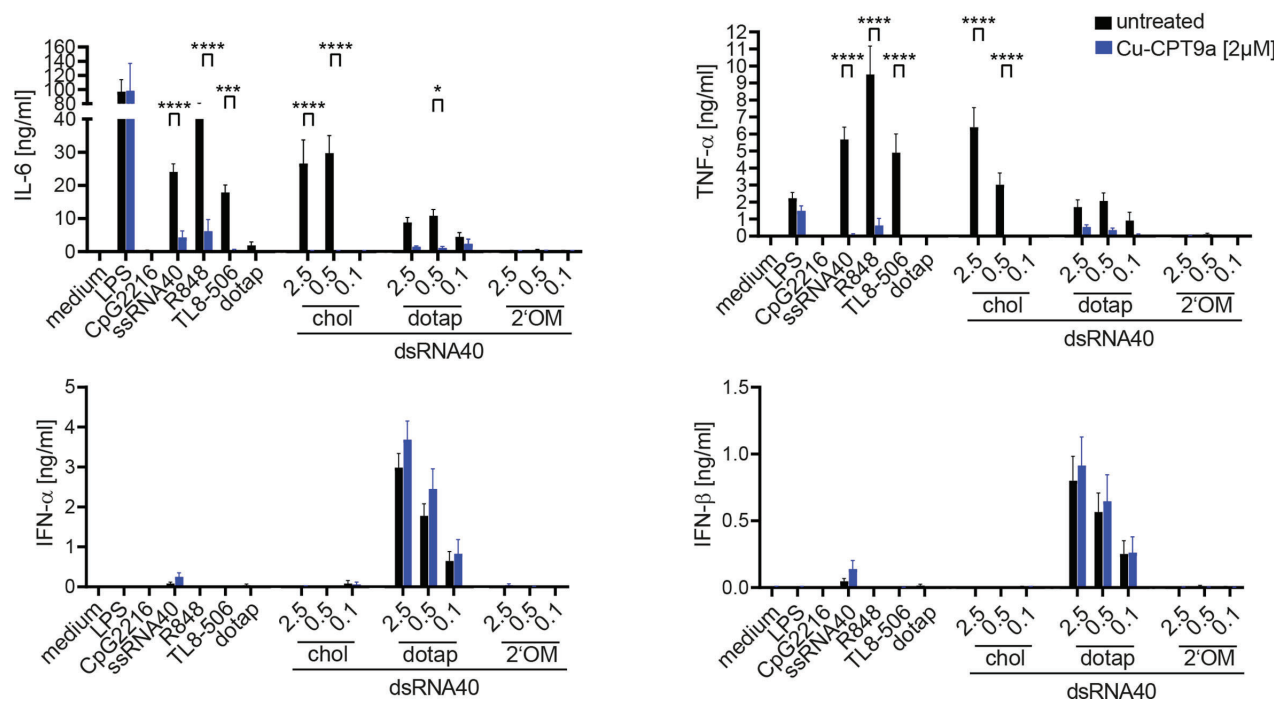

FIGURE 2 | (A) Wt BMDMs were stimulated with $0.5 \mu \mathrm{M}$ ssRNA40-chol or ssRNA40 complex to DOTAP or $0.5 \mu \mathrm{M}$ dsRNA40-chol, dsRNA40 complexed to DOTAP and dsRNA40-chol-2'OM. Medium, CpG2216 (1 $\mu \mathrm{M})$, LPS $(1 \mathrm{\mu g} / \mathrm{ml})$ and DOTAP served as controls. Supernatants were taken after $20 \mathrm{~h}$ of incubation and cytokine production was analyzed by ELISA. Data were calculated from three individual experiments. Bars indicate mean + SEM. Unpaired t-test was used for comparison of ssRNA40 samples while one-way-ANOVA with Sidak's post-hoc test was used for comparison of dsRNA40 samples. ${ }^{\star} \mathrm{p}<0.05,{ }^{* *} \mathrm{p}<0.01$, ${ }_{* \star \star \star} \mathrm{p}<0.0001$. (B) Purified human Monocytes were isolated via MACS separation, incubated with (blue bars) or without (black bars) $2 \mu \mathrm{M}$ of the TLR8 inhibitor Cu-CPT9a and stimulated with dsRNA40-chol, dsRNA40 complexed to DOTAP and dsRNA40-chol-2'OM at $2.5 \mu \mathrm{M}, 0.5 \mu \mathrm{M}$ and $0.1 \mu \mathrm{M}$. Medium, CpG2216 $(1 \mu \mathrm{M})$, LPS $(1 \mu \mathrm{g} / \mathrm{ml})$, ssRNA/DOTAP $(2,5 \mu \mathrm{M})$, R848 $(2,5 \mu \mathrm{g} / \mathrm{mll})$, TL8-506 $(60 \mathrm{nM})$ and DOTAP served as controls. Supernatants were harvested after $20 \mathrm{~h}$ of incubation and IL-6, TNF- $\alpha$, IFN- $\alpha$, IFN- $\beta$ were analyzed by ELISA. Data were calculated from at least three (for TL8-506) to six independent experiments. Bars indicate mean + SEM. ${ }^{*} p<0.05,{ }^{\star \star \star} p<0.001,{ }^{\star \star * \star} p<0.0001$; two-way ANOVA with Sidak's post-hoc test.

monocytes. As a negative control dsRNA40-chol with alternating 2'O-ribose-methylation did not show any immunostimulatory activity (Figure 2B).

\section{Physicochemical Characterization of dsRNA40-Chol}

Atomic force microscopy (AFM) measurements indicated spherically round shaped dsRNA40-chol nanoparticles formed by self-assembly (Figures 3A-C). The LDA analysis of the nanoparticles exhibit a strong negative zeta potential of $-29.4 \pm 5.5 \mathrm{mV}$ (Figure 3D). The particles diameters result from the evaluation of images in the measured height mode. Three particle size fractions are visible by $\operatorname{AFM}(42.3 \pm 4.8 \mathrm{~nm}$ about $30 \%, 81.5 \pm 7.4 \mathrm{~nm}$ about $65 \%$ and a small fraction of $198 \pm 11.5 \mathrm{~nm}$ ) with an average diameter of $79.5 \pm 9.4 \mathrm{~nm}$. The prepared particles exhibited hydrodynamic diameters (by PCS) of $41.7 \pm 6.4 \mathrm{~nm}, 122.4 \pm 11.3 \mathrm{~nm}$ and $190.1 \pm 17.4 \mathrm{~nm}$ (distribution by volume, Figure 3E) with a mean diameter $\mathrm{z}$-AvE of $97.8 \pm 16.5$ $\mathrm{nm}$, which are in principle in accordance with the AFM measurements. It should however be noted that the differences in size arising from the PCS and AFM measurements is because the hydrodynamic diameter obtained in aqueous conditions while the latter is measured at atmospheric conditions.

\section{dsRNA40-Chol Nanoparticles Are Efficiently Taken Up by Different Immune Cells}

To address the uptake of self-assembled dsRNA40-chol nanoparticles by immune cells, we incubated human and murine pDC and human monocytes with Alexa488-labeled dsRNA40-chol, Alexa488-labeled dsRNA40 complexed to 


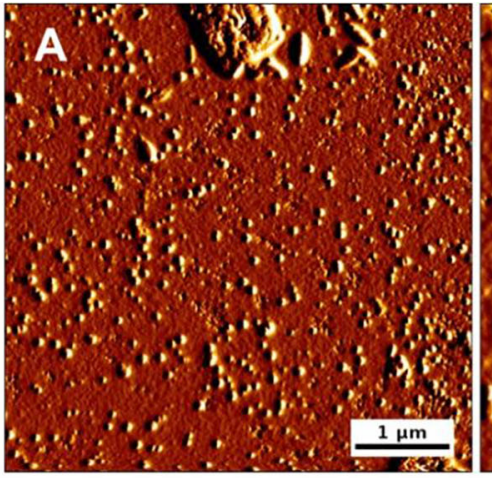

Zeta Potential Distrbution

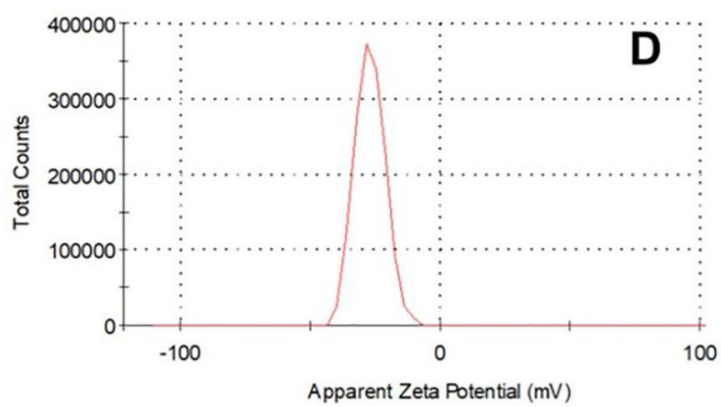

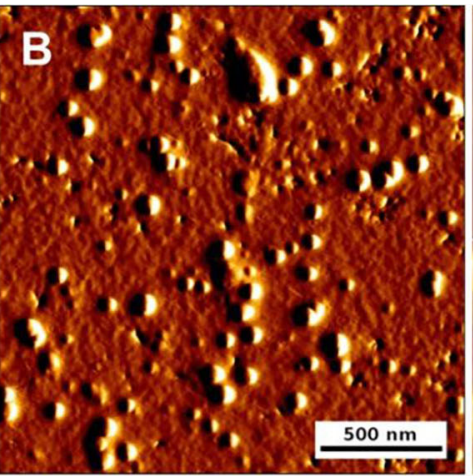

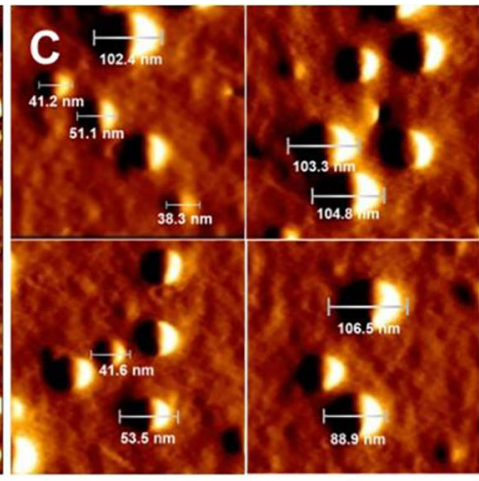

Size Distribution by Volume

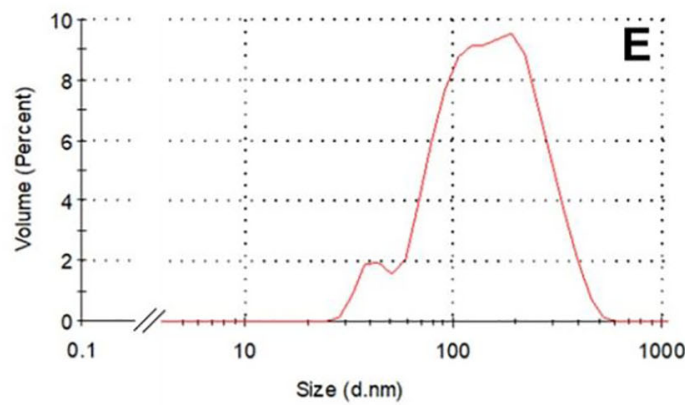

FIGURE 3 | dsRNA40-chol forms nanoparticles by self-assembly. (A-C) Morphology of representative dsRNA40-chol nanoparticles shown by atomic force microscopy. All micrographs are presented in amplitude mode. (D) represents the LDA zeta potential measurements and (E) the size distribution and the diameter of the particles.

DOTAP or Alexa488-labeled dsRNA40 without any conjugation or complex formation. Cells were analyzed by confocal laser scanning microscopy and FACS. Importantly, dsRNA40-chol was taken up efficiently by human pDC when compared to naked dsRNA40 or RNA40 complexed to DOTAP (Figure 4A). Similarly, quantification of RNA uptake by murine and human pDCs as well as human monocytes using flow cytometry showed significantly improved uptake of dsRNA40-chol compared to naked or complexed dsRNA40 (Figure 4B).

\section{dsRNA40-Chol Nanoparticles Induce Proinflammatory Cytokines in Purified Human Monocytes and Promote $\mathrm{T}_{\mathrm{H} 1}$ and $\mathrm{T}_{\mathrm{FH}}$ Cell Responses}

To study the adjuvant potential of ssRNA40-chol or dsRNA40chol nanoparticles we set up an in vitro $\mathrm{T}$-cell differentiation assay where CD3- and CD28-activated $\mathrm{CD}^{+} \mathrm{T}$ cells were incubated with supernatant from stimulated monocytes containing differentiation-promoting cytokines (26). We focused in particular on the differentiation of IFN- $\gamma$-producing $\mathrm{T}$ helper type 1 cells $\left(\mathrm{T}_{\mathrm{H} 1}\right)$ and IL-21-producing $\mathrm{T}$ follicular helper cells $\left(\mathrm{T}_{\mathrm{FH}}\right) \cdot \mathrm{T}_{\mathrm{H} 1}$ cell play a fundamental role in the host defense response against intracellular pathogens, whereas $\mathrm{T}_{\mathrm{FH}}$ cells are important regulators of the germinal-center response and humoral immunity by interacting with B-cells (27). Accordingly, we stimulated purified human monocytes with ssRNA-chol or
dsRNA-chol and used the supernatants for T cell differentiation. The analysis of the supernatants showed that dsRNA40-chol induced IL-6, TNF- $\alpha$, IL- $1 \beta$ and IL-12p70. IL-6 production was higher at $0.5 \mu \mathrm{M}$ of dsRNA40-chol in comparison to $2.5 \mu \mathrm{M}$, whereas TNF- $\alpha$, IL-1 $\beta$ and IL-12p70 secretion was more prominent at $2.5 \mu \mathrm{M}$ (Figure $\mathbf{4 A}$ ). Interestingly, ssRNA40-chol showed some cytokine (e.g. IL-6 and TNF- $\alpha$ ) induction in human monocytes (Figure 5A) although less potent than dsRNA40-chol. Again, we could not detect any cytokine production induced by dsRNA40-chol with alternating 2'O-ribose-methylation at the antisense strand (Figure 5A). To determine the contribution of RNA40-chol induced innate immunostimulation to human T cell differentiation, we analyzed whether supernatants harvested from RNA40-chol stimulated monocytes had any influence on T cells, in particular the differentiation of IFN- $\gamma$-producing $\mathrm{T}_{\mathrm{H} 1}$ and IL21-producing $\mathrm{T}_{\mathrm{FH}}$ cells. Indeed, $\mathrm{CD}^{+} \mathrm{T}$ cells showed significantly increased intracellular IFN- $\gamma$ expression if activated by CD3 and CD28 in presence of supernatants of purified monocytes stimulated with $2.5 \mu \mathrm{M}$ of ssRNA40-chol and $2.5 \mu \mathrm{M}$ or $0.5 \mu \mathrm{M}$ of dsRNA40-chol (Figure 5B). Additionally, monocytes stimulated with $2.5 \mu \mathrm{M}$ of dsRNA40-chol were able to induce expression of the transcription factor Bcl-6, which is important for $\mathrm{T}_{\mathrm{FH}}$ cell development $(28,29)$ and IL-21 in $\mathrm{CD}^{+} \mathrm{T}$ cells (Figure 5B). In summary, ssRNA40-chol promotes $\mathrm{T}_{\mathrm{H} 1}$ cell differentiation, whereas dsRNA40-chol promotes $\mathrm{T}_{\mathrm{H} 1}$ as well as $\mathrm{T}_{\mathrm{FH}}$ cell differentiation. 

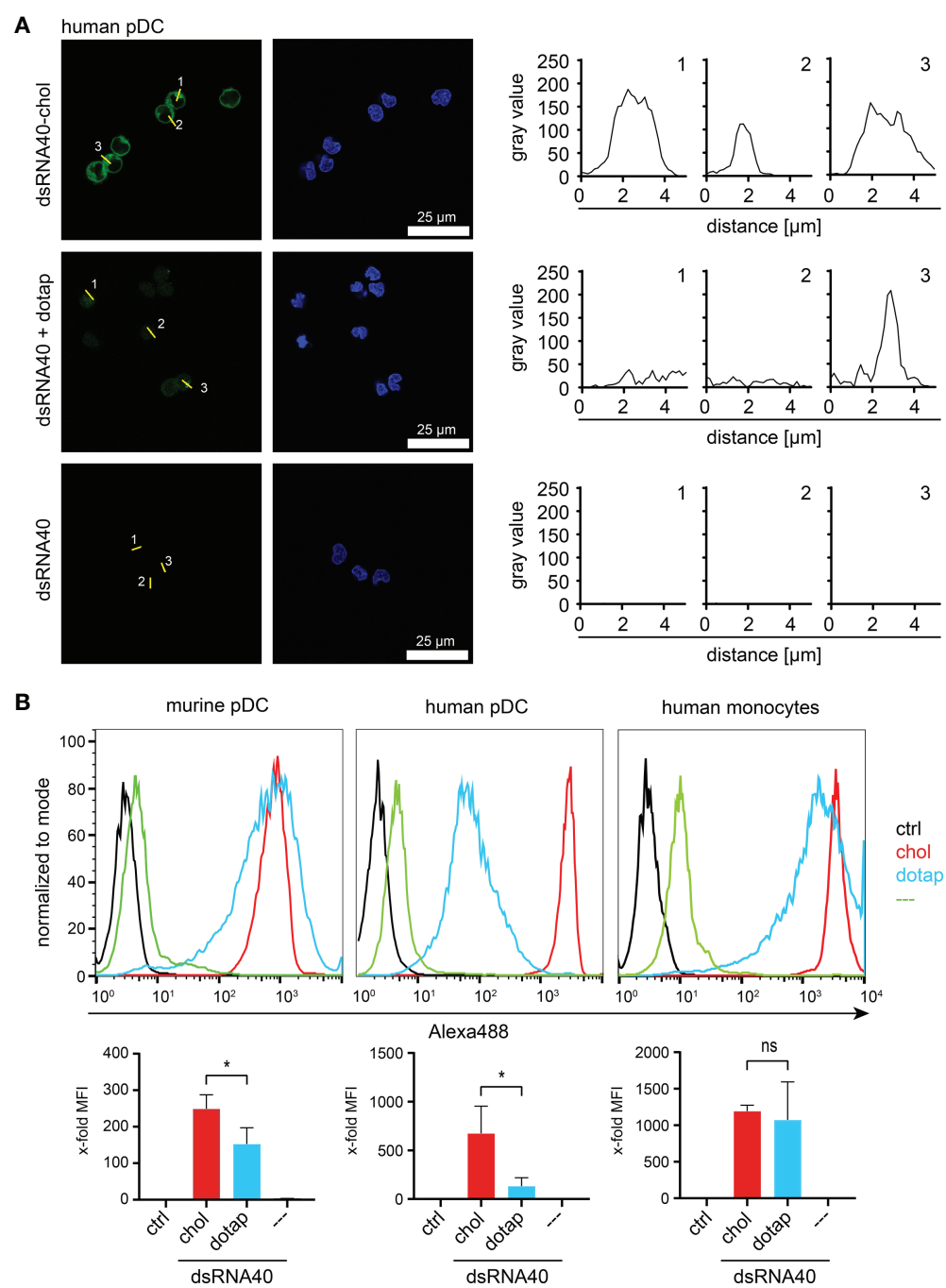

FIGURE 4 | dsRNA40-chol nanoparticles are efficiently taken up by different immune cells. (A) Purified human pDC were incubated with 0.5 4 M of Alexa488-labeled dsRNA40-chol, dsRNA40 complexed to DOTAP or dsRNA40. Cells were extensively washed and fixed after $1.5 \mathrm{~h}$ of incubation and analyzed by confocal laser scanning microscopy. RNA40 is shown in green and DAPI-stained nuclei in blue (left panel, scale bar $25 \mu \mathrm{m}$ ). In the right panel, the fluorescence intensity profile is plotted as grayscale (fluorescence intensity) versus distance (in $\mu \mathrm{M}$ ) for individual cells. For every cell stimulation condition, 3 cells were marked by a line (numbered from 1 to 3) and corresponding fluorescence profile (numbered from 1 to 3) plotted along this line. (B) Murine Flt3L-DC (B220+/CD11C+ pDC), purified human pDC and monocytes were incubated as described in (B) and uptake of RNA40 was analyzed by flow cytometry. Histograms depict one representative experiment out of three independent experiments. Bar charts combine median fluorescent intensities (MFI) of three independent experiments. Values were normalized to ctrl. Bars indicate mean + SEM. ns: not significant, " $\mathrm{p}<0.05$; one-way ANOVA with Sidak's post-hoc test.

\section{DISCUSSION}

Immunostimulation of the innate immune system with subsequent activation and tuning of the adaptive immune response relies on activation of germline-encoded PRR by pathogen-associated molecular patterns (PAMP). Amongst other PRR, ssRNA as well as dsRNA have been reported to stimulate the immune system via TLR7 and $\operatorname{TLR} 8(1,2)$. More precisely, both TLR recognize RNA degradation products present as nucleosides and oligoribonucleotides. TLR7 detects guanosine and uridine moieties in ssRNA (4) and TLR8 recognizes uridine and in addition a short oligonucleotide created by RNase T2 and/or RNase 2 (5-7). Cellular uptake of nucleic acids into the endosomal compartment, where TLR that recognize nucleic acids are localized $(30,31)$, has to be facilitated to analyze nucleic acid-induced immune responses. Cells are usually transfected with liposome-nucleic acid complex consisting of nucleic acid molecules complexed to transfection reagents like DOTAP, because otherwise nucleic acids hardly interact with negatively charged cell membranes and are highly prone to degradation by RNases or DNases before they reach the endosomal compartment. Common transfection strategies 

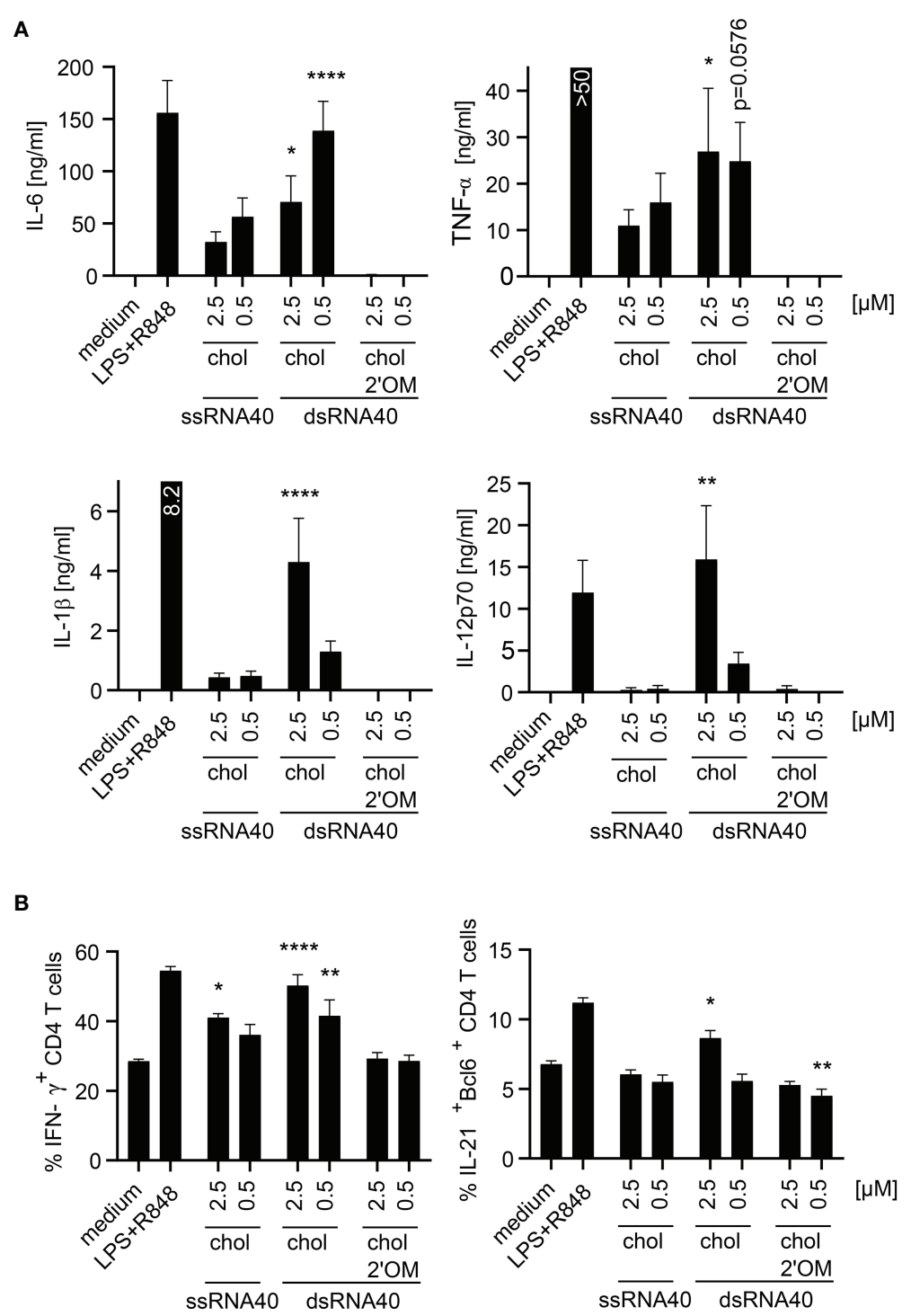

FIGURE 5 | dsRNA40-chol nanoparticles induce proinflammatory cytokines in purified human monocytes and promotes $\mathrm{T}_{\mathrm{H} 1}$ and $\mathrm{T}_{\mathrm{FH}}$ cell responses. (A) Purified human monocytes were stimulated with ssRNA40-chol, dsRNA40-chol and dsRNA40-chol-2'OM at $2.5 \mu \mathrm{M}$ and $0.5 \mu \mathrm{M}$. Medium and LPS (1 $\mu \mathrm{g} / \mathrm{ml}$ ) in combination with R848 $(2.5 \mu \mathrm{g} / \mathrm{ml})$ served as controls. Supernatants were harvested after $20 \mathrm{~h}$ of incubation and cytokine induction was analyzed by ELISA. Data were calculated from six individual donors. Bars indicate mean + SEM. ${ }^{*} \mathrm{p}<0.05,{ }^{* *} \mathrm{p}<0.01,{ }^{\star \star \star *} \mathrm{p}<0.0001$; one-way ANOVA with Dunnett's post-hoc test. (B) Purified human $\mathrm{CD}^{+} \mathrm{T}$ cells were restimulated with anti-CD3 and anti-CD28 and incubated with conditioned monocyte supernatant [monocytes were stimulated as described in (A)]. Cells were incubated for five days before they were analyzed by flow cytometry. Data were calculated from four individual donors. Bars indicate mean + SEM. ${ }^{\star} \mathrm{p}<0.05,{ }^{\star \star} \mathrm{p}<0.01,{ }^{\star \star \star \star} \mathrm{p}<0.0001$; one-way ANOVA with Dunnett's post-hoc test.

require an initial liposome-nucleic acid complex formation step prior to adding the immune activator/TLRL to target cells, which then enters the cells via endocytosis-like processes $(32,33)$ or by temperature-independent transport mechanisms through cell membranes (33).

Therefore, we focused on characterization of a simple system that does not depend on the requirement of initial liposomenucleic acid complex formation to activate the immune system by the TLR7 and TLR8 ligand RNA40. We analyzed the immunostimulation in plasmacytoid dendritic cells induced by
ssRNA40-chol containing a phosphodiester backbone but could not detect significant amounts of IFN- $\alpha$, IL-6, TNF- $\alpha$ and CXCL10 in comparison to ssRNA40 complexed to DOTAP. This outcome was most likely due to increased susceptibility of ssRNA40-chol to RNAse-digestion. Forsbach et al. (11) have shown that cholesterol-conjugated ssORN with highly nuclease resistant phosphorothioate nucleotide linkage activates human PBMC and murine spleen DC to produce IFN- $\alpha$ and TNF- $\alpha$. Furthermore, they described that mice responded with CXCL10 secretion after i.v. injection of ssORN-chol containing a 
phosphorothioate backbone (11). However, dsRNA conjugated to cholesterol was not tested. In addition, it has been described for antisense oligonucleotide delivery that it may be important to cleave the phosphodiester between cholesterol and antisense oligonucleotide intracellularly $(25,26)$. For that reason, cholesterol-conjugation with phosphorothioate RNA40 may be less active for recognition by PRR. Additionally, toxicities in association with phosphorothioate linkage have been reported (27). Furthermore, we hypothesized that RNase-digest of dsRNA40-chol leads to higher amounts of TLR7/TLR8 stimulatory fragments in contrast to ssRNA40-chol which could be digested in total. Therefore, we investigated the immunostimulatory potential of dsRNA40-chol instead and detected that dsRNA40-chol was superior to dsRNA40 complexed to DOTAP in its ability to induce IFN- $\alpha$ and TNF$\alpha$ in murine Flt3L-DC. IL- 6 and CXCL10 secretion was also induced by dsRNA40-chol and cytokine induction was strictly dependent on TLR7 in murine Flt3L-DC. In contrast, human monocytes responded strongly with IL- 6 and TNF- $\alpha$ production to dsRNA40-chol stimulation in a strictly TLR8-dependent manner. Since TLR8 in mice is non-responsive to RNA ligands (17, 34, 35), murine bone marrow derived macrophages (BMDM) were not stimulated by dsRNA40-chol. In contrast, DOTAP-complexed dsRNA40 induced strong type I interferon production in a TLR8-independent fashion suggesting that TLR7 in monocytes is activated by this formulation. Our results contrast a recent study by de Marcken $\mathrm{M}$ et al. (36) that describes type I interferon production by TLR8 and not TLR7 in human monocytes. The reason for this discrepancy is currently unknown and may be dependent on the different systems used.

We further characterized the dsRNA-chol formulation by atomic force microscopy and photon correlation spectroscopy. This analysis showed, that dsRNA40-chol forms nanoparticles of three particle size fractions. Currently, we do not know why dsRNA40-chol generates heterogeneous nanoparticles, but for further advancing this formulation to a clinical application, it would be necessary to control particle size and formation. In addition, this self-assembly of dsRNA40-chol nanoparticles is somewhat reminiscent of the formation of immune stimulating complexes (ISCOMs) that are spherical cage-like nanoparticles consisting of the saponin adjuvant Quil A, cholesterol, phospholipids and antigens (37). However, the dsRNA40-chol nanoparticles are devoid of antigen and future experiments should focus on integrating antigen into these nanoparticles. Thorough analysis of the dsRNA-chol nanoparticles demonstrated that they are taken up by murine $\mathrm{pDC}$, human $\mathrm{pDC}$ and human monocytes and are efficiently internalized. Within the human system, we found that dsRNA40-chol lead to secretion of IFN- $\alpha$ in purified pDC. Purified human monocytes responded with IL-6, TNF- $\alpha$, IL-1 $\beta$ and IL-12p70 secretion. Comparison of murine Flt3L-DC/human pDC with human monocytes showed that higher amounts of dsRNA40-chol nanoparticles are needed to activate monocytes. These observations are confirmed by studies by Rettig et al. (38). They found that human pDC are efficient in taking up immunostimulatory protamine-ssRNA nanoparticles and respond with IFN- $\alpha$ secretion. Furthermore, they could show that human monocytes are able to internalize nano- as well as microparticles and exhibit a higher threshold of activation in contrast to pDC. Interestingly, human monocytes produced IL-6 and TNF- $\alpha$ in response to ssRNA40-chol as well. This could be due to higher phagocytotic activity of monocytes in comparison to $\mathrm{pDC}$. Our finding that dsRNA40-chol induces IL-1 $\beta$ in human monocytes indicates that dsRNA40-chol cannot only activate murine TLR7 but also activates human TLR8. This is supported by investigations of human monocytes showing that IL-1 $\beta$ secretion after HIV infection depends mainly on TLR8 and only to minimal extent on TLR7 (39) and by data demonstrating that TLR8, and not TLR7, activates the NLRP3 inflammasome resulting in IL-1 $\beta$ secretion (40). This observation is further supported by the fact that TLR8 is in contrast to TLR7 highly expressed in human monocytes $(26,41)$. Another aspect, which supports TLR8 activation by RNA40-chol is that TLR 8 and not TLR7 activation has been demonstrated to promote $\mathrm{T}_{\mathrm{H} 1}$ cells (36), which is in line with our data showing that dsRNA40-chol and ssRNA40-chol drove $\mathrm{T}_{\mathrm{H} 1}$ cell differentiation. In addition, dsRNA40-chol induced $\mathrm{T}_{\mathrm{FH}}$ cell differentiation, which depends like $\mathrm{T}_{\mathrm{H} 1}$ cell differentiation on IL-12p70 $(26,42,43)$ and secretion of IL-12p70 by human monocytes depends on TLR8 and is non-redundant with other TLR $(44,45)$. Of note, it has just recently been reported that activation of TLR 8 by dsRNA40 requires RNase 2 as well as RNase T2 and both RNases cooperate to cleave dsRNA substrates (7).

To exclude an immunostimulatory potential by cholesterol, we used dsRNA40-chol with alternating 2'O-ribose-methylation, which renders immune activating RNA non-stimulatory (19-25) because it is neither digested by RNase 2 nor RNase T2 (7), and found no stimulatory effect in all cell types tested. Additionally, in vivo studies showed that chol-conjugated nucleic acids are taken up into several tissues $(9,10,46)$. The most important one is the liver since highdensity lipoprotein (HDL) and especially low-density lipoprotein (LDL) are important delivery proteins for cholesterol-conjugated nucleic acids $(9,10)$. Given that observation in addition to the knowledge that IFN- $\alpha$ induction by TLR7 agonists is of high interest during chronic hepatitis B virus infection (47-49), conjugation of TLR7-ligands to cholesterol could be a therapeutical approach. Since cholesterol-conjugated nucleic acids are predominantly delivered to liver, kidney and spleen (46), all belonging to primary clearance tissues, these organs are potential targets for dsRNA40-chol as TLR7/8 agonist.

In summary, we investigated the immunostimulatory potential of cholesterol-conjugated dsRNA40 and demonstrated efficient internalization and cytokine induction in various immune cells with the potential of driving $\mathrm{T}$ cell differentiation. Therefore, dsRNA40-chol may be a simple and potent adjuvant for future clinical use without the need for separate carriers such as liposomes.

\section{DATA AVAILABILITY STATEMENT}

The raw data supporting the conclusions of this article will be made available by the authors, without undue reservation. 


\section{ETHICS STATEMENT}

The local ethics committees of Justus-Liebig-University Giessen and Philipps-University Marburg reviewed and approved the use of human blood samples for this study and written informed consent was obtained from the blood donors. For experiments with murine immune cells, mice were sacrificed and tissue/organs removed. These experiments were performed in accordance with the National German welfare law $\$ 4(3)$ TierSchG and $\$ 2$ and Annex 2 (TierSchVerV) of the National Order for the use of animals in research and do not need the approval by a local ethics committee. According to the regulations, the number of mice used was reported to the animal welfare officer of the Philipps-University Marburg.

\section{AUTHOR CONTRIBUTIONS}

H-LO, IL, JS, JD, KE, and UB performed experiments. H-LO, LES, UB, AK, and SB designed the experiments. H-LO, AK, JS, LES, UB, and SB analyzed the data. H-LO and SB wrote the manuscript. H-LO and SB conceived and supervised the study.

\section{REFERENCES}

1. Heil F, Hemmi H, Hochrein H, Ampenberger F, Kirschning C, Akira S, et al. Species-Specific Recognition of Single-Stranded RNA via Toll-Like Receptor 7 and 8. Science (2004) 303:1526-9. doi: 10.1126/science. 1093620

2. Hornung V, Guenthner-Biller M, Bourquin C, Ablasser A, Schlee M, Uematsu $S$, et al. Sequence-Specific Potent Induction of IFN-Alpha by Short Interfering RNA in Plasmacytoid Dendritic Cells Through TLR7. Nat Med (2005) 11:263-70. doi: 10.1038/nm1191

3. Barbalat R, Ewald SE, Mouchess ML, Barton GM. Nucleic Acid Recognition by the Innate Immune System. Annu Rev Immunol (2011) 29:185-214. doi: 10.1146/annurev-immunol-031210-101340

4. Zhang Z, Ohto U, Shibata T, Krayukhina E, Taoka M, Yamauchi Y, et al. Structural Analysis Reveals That Toll-Like Receptor 7 Is a Dual Receptor for Guanosine and Single-Stranded RNA. Immunity (2016) 45:737-48. doi: 10.1016/j.immuni.2016.09.011

5. Tanji H, Ohto U, Shibata T, Taoka M, Yamauchi Y, Isobe T, et al. Toll-Like Receptor 8 Senses Degradation Products of Single-Stranded RNA. Nat Struct Mol Biol (2015) 22:109-15. doi: 10.1038/nsmb.2943

6. Greulich W, Wagner M, Gaidt MM, Stafford C, Cheng Y, Linder A, et al. TLR8 Is a Sensor of RNase T2 Degradation Products. Cell (2019) 179:126475.e13. doi: 10.1016/j.cell.2019.11.001

7. Ostendorf T, Zillinger T, Andryka K, Schlee-Guimaraes TM, Schmitz S, Marx S, et al. Immune Sensing of Synthetic, Bacterial, and Protozoan RNA by TollLike Receptor 8 Requires Coordinated Processing by RNase T2 and RNase 2. Immunity (2020) 52:591-605.e6. doi: 10.1016/j.immuni.2020.03.009

8. Gamper HB, Reed MW, Cox T, Virosco JS, Adams AD, Gall AA, et al. Facile Preparation of Nuclease Resistant 3' Modified Oligodeoxynucleotides. Nucleic Acids Res (1993) 21:145-50. doi: 10.1093/nar/21.1.145

9. Soutschek J, Akinc A, Bramlage B, Charisse K, Constien R, Donoghue M, et al. Therapeutic Silencing of an Endogenous Gene by Systemic Administration of Modified siRNAs. Nature (2004) 432:173-8. doi: 10.1038/nature03121

10. Wolfrum C, Shi S, Jayaprakash KN, Jayaraman M, Wang G, Pandey RK, et al. Mechanisms and Optimization of In Vivo Delivery of Lipophilic siRNAs. Nat Biotechnol (2007) 25:1149-57. doi: 10.1038/nbt1339

11. Forsbach A, Samulowitz U, Völp K, Hofmann H-P, Noll B, Tluk S, et al. Dual or Triple Activation of TLR7, TLR8, and/or TLR9 by Single-Stranded Oligoribonucleotides. Nucleic Acid Ther (2011) 21:423-36. doi: 10.1089/nat. 2011.0323
All authors contributed to the article and approved the submitted version.

\section{FUNDING}

This work was funded by the German Center for Infection Research (TTU 09.806 to SB); the Deutsche Forschungsgemeinschaft (DFG, German Research Foundation) Project-ID 369799452 TRR237 A02 to SB and Project-ID 114933180 TR84 - C10 to SB and LES.

\section{ACKNOWLEDGMENTS}

We thank H. Hackstein and G. Bein, Institute for Clinical Immunology and Transfusion Medicine, Justus-Liebig-University Giessen, for providing human buffy coats. Parts of this work have been investigated within the doctoral thesis "Die Rolle von RNA/ DNA Hybriden in der angeborenen Immunantwort und das Potential von cholesterolkonjugierter RNA als Adjuvans" written by H-LO.

12. Rattanakiat S, Nishikawa M, Takakura Y. Self-Assembling CpG DNA Nanoparticles for Efficient Antigen Delivery and Immunostimulation. Eur J Pharm Sci (2012) 47:352-8. doi: 10.1016/j.ejps.2012.06.015

13. Alahari SK, Dean NM, Fisher MH, Delong R, Manoharan M, Tivel KL, et al. Inhibition of Expression of the Multidrug Resistance-Associated PGlycoprotein of by Phosphorothioate and 5' Cholesterol-Conjugated Phosphorothioate Antisense Oligonucleotides. Mol Pharmacol (1996) 50:808-19.

14. Bijsterbosch MK, Rump ET, de VRL, Dorland R, van Veghel R, KL T, et al. Modulation of Plasma Protein Binding and In Vivo Liver Cell Uptake of Phosphorothioate Oligodeoxynucleotides by Cholesterol Conjugation. Nucleic Acids Res (2000) 28:2717-25. doi: 10.1093/nar/28.14.2717

15. Desjardins J, Mata J, Brown T, Graham D, Zon G, Iversen P. CholesterylConjugated Phosphorothioate Oligodeoxynucleotides Modulate CYP2B1 Expression In Vivo. J Drug Target (1995) 2:477-85. doi: 10.3109/ 10611869509015917

16. DiFiglia M, Sena-Esteves M, Chase K, Sapp E, Pfister E, Sass M, et al. Therapeutic Silencing of Mutant Huntingtin With siRNA Attenuates Striatal and Cortical Neuropathology and Behavioral Deficits. Proc Natl Acad Sci U S A (2007) 104:17204-9. doi: 10.1073/pnas.0708285104

17. Hemmi H, Kaisho T, Takeuchi O, Sato S, Sanjo H, Hoshino K, et al. Small AntiViral Compounds Activate Immune Cells via the TLR7 MyD88-Dependent Signaling Pathway. Nat Immunol (2002) 3:196-200. doi: 10.1038/ni758

18. Sitterberg J, Ozcetin A, Ehrhardt C, Bakowsky U. Utilising Atomic Force Microscopy for the Characterisation of Nanoscale Drug Delivery Systems. Eur J Pharm Biopharm (2010) 74:2-13. doi: 10.1016/j.ejpb.2009.09.005

19. Hamm S, Latz E, Hangel D, Müller T, Yu P, Golenbock D, et al. Alternating 2'O-Ribose Methylation is a Universal Approach for Generating Non-Stimulatory siRNA by Acting as TLR7 Antagonist. Immunobiology (2010) 215:559-69. doi: 10.1016/j.imbio.2009.09.003

20. Karikó K, Buckstein M, Ni H, Weissman D. Suppression of RNA Recognition by Toll-Like Receptors: The Impact of Nucleoside Modification and the Evolutionary Origin of RNA. Immunity (2005) 23:165-75. doi: 10.1016/ j.immuni.2005.06.008

21. Robbins M, Judge A, Liang L, McClintock K, Yaworski E, MacLachlan I. 2'-OMethyl-Modified RNAs Act as TLR7 Antagonists. Mol Ther (2007) 15:1663-9. doi: 10.1038/sj.mt.6300240

22. Sioud M, Furset G, Cekaite L. Suppression of Immunostimulatory siRNADriven Innate Immune Activation by 2'-Modified RNAs. Biochem Biophys Res Commun (2007) 361:122-6. doi: 10.1016/j.bbrc.2007.06.177 
23. Gehrig S, Eberle M-E, Botschen F, Rimbach K, Eberle F, Eigenbrod T, et al. Identification of Modifications in Microbial, Native tRNA That Suppress Immunostimulatory Activity. J Exp Med (2012) 209:225-33. doi: 10.1084/jem.20111044

24. Jöckel S, Nees G, Sommer R, Zhao Y, Cherkasov D, Hori H, et al. The 2'-OMethylation Status of a Single Guanosine Controls Transfer RNA-Mediated Toll-Like Receptor 7 Activation or Inhibition. J Exp Med (2012) 209:235-41. doi: 10.1084/jem.20111075

25. Jung S, von Thülen T, Laukemper V, Pigisch S, Hangel D, Wagner H, et al. A Single Naturally Occurring 2'-O-Methylation Converts a TLR7- and TLR8Activating RNA Into a TLR8-Specific Ligand. PLoS One (2015) 10:e0120498. doi: 10.1371/journal.pone.0120498

26. Ugolini M, Gerhard J, Burkert S, Jensen KJ, Georg P, Ebner F, et al. Recognition of Microbial Viability via TLR8 Drives TFH Cell Differentiation and Vaccine Responses. Nat Immunol (2018) 19:386-96. doi: 10.1038/s41590-018-0068-4

27. Tuzlak S, Dejean AS, Iannacone M, Quintana FJ, Waisman A, Ginhoux F, et al. Repositioning TH Cell Polarization From Single Cytokines to Complex Help. Nat Immunol (2021) 22:1210-7. doi: 10.1038/s41590-021-01009-w

28. Nurieva RI, Chung Y, Martinez GJ, Yang XO, Tanaka S, Matskevitch TD, et al. Bcl6 Mediates the Development of T Follicular Helper Cells. Science (2009) 325:1001-5. doi: 10.1126/science.1176676

29. Johnston RJ, Poholek AC, DiToro D, Yusuf I, Eto D, Barnett B, et al. Bcl6 and Blimp-1 are Reciprocal and Antagonistic Regulators of T Follicular Helper Cell Differentiation. Science (2009) 325:1006-10. doi: 10.1126/science.1175870

30. Brubaker SW, Bonham KS, Zanoni I, Kagan JC. Innate Immune Pattern Recognition: A Cell Biological Perspective. Annu Rev Immunol (2015) 33:25790. doi: 10.1146/annurev-immunol-032414-112240

31. Schlee M, Hartmann G. Discriminating Self From Non-Self in Nucleic Acid Sensing. Nat Rev Immunol (2016) 16:566-80. doi: 10.1038/nri.2016.78

32. Zabner J, Fasbender AJ, Moninger T, Poellinger KA, Welsh MJ. Cellular and Molecular Barriers to Gene Transfer by a Cationic Lipid. J Biol Chem (1995) 270:18997-9007. doi: 10.1074/jbc.270.32.18997

33. Marchini C, Pozzi D, Montani M, Alfonsi C, Amici A, Candeloro De Sanctis S, et al. Role of Temperature-Independent Lipoplex-Cell Membrane Interactions in the Efficiency Boost of Multicomponent Lipoplexes. Cancer Gene Ther (2011) 18:543-52. doi: 10.1038/cgt.2011.12

34. Forsbach A, Nemorin J-G, Montino C, Müller C, Samulowitz U, Vicari AP, et al. Identification of RNA Sequence Motifs Stimulating Sequence-Specific TLR8-Dependent Immune Responses. J Immunol (2008) 180:3729-38. doi: 10.4049/jimmunol.180.6.3729

35. Gorden KK, Qiu XX, Binsfeld CC, Vasilakos JP, Alkan SS. Cutting Edge: Activation of Murine TLR8 by a Combination of Imidazoquinoline Immune Response Modifiers and polyT Oligodeoxynucleotides. J Immunol (2006) 177:6584-7. doi: 10.4049/jimmunol.177.10.6584

36. de Marcken M, DhaliwalK, DanielsenAC, Gautron AS, Dominguez-Villar M. TLR7 and TLR8 Activate Distinct Pathways in Monocytes During RNA Virus Infection. Sci Signal (2019) 12. doi: 10.1126/scisignal.aaw1347

37. Kersten GF, Crommelin DJ. Liposomes and ISCOMs. Vaccine (2003) 21:91520. doi: 10.1016/s0264-410x(02)00540-6

38. Rettig L, Haen SP, Bittermann AG, von BoehmerL, CurioniA, Krämer SD, et al. Particle Size and Activation Threshold: A New Dimension of Danger Signaling. Blood (2010) 115:4533-41. doi: 10.1182/blood-2009-11-247817

39. Chattergoon MA, Latanich R, Quinn J, Winter ME, Buckheit RW, Blankson JN, et al. HIV and HCV Activate the Inflammasome in Monocytes and Macrophages via Endosomal Toll-Like Receptors Without Induction of Type 1 Interferon. PLoS Pathog (2014) 10:e1004082. doi: 10.1371/journal.ppat. 1004082
40. Vierbuchen T, Bang C, Rosigkeit H, Schmitz RA, Heine H. The HumanAssociated Archaeon Methanosphaera Stadtmanae Is Recognized Through Its RNA and Induces TLR8-Dependent NLRP3 Inflammasome Activation. Front Immunol (2017) 8:1535. doi: 10.3389/fimmu.2017.01535

41. Hornung V, Rothenfusser S, Britsch S, Krug A, Jahrsdörfer B, Giese T, et al. Quantitative Expression of Toll-Like Receptor 1-10 mRNA in Cellular Subsets of Human Peripheral Blood Mononuclear Cells and Sensitivity to CpG Oligodeoxynucleotides. J Immunol (2002) 168:4531-7. doi: 10.4049/ jimmunol.168.9.4531

42. Hsieh CS, Macatonia SE, Tripp CS, Wolf SF, O’Garra A, Murphy KM. Development of TH1 CD4+ T Cells Through IL-12 Produced by ListeriaInduced Macrophages. Science (1993) 260:547-9. doi: 10.1126/science.8097338

43. Perez VL, Lederer JA, Carniel E, Lichtman A, Abbas AK. The Role of IL12 in Helper T-Cell Differentiation. Res Immunol (1995) 146:477-80. doi: 10.1016/ 0923-2494(96)83019-7

44. Ablasser A, Poeck H, Anz D, Berger M, Schlee M, Kim S, et al. Selection of Molecular Structure and Delivery of RNA Oligonucleotides to Activate TLR7 Versus TLR8 and to Induce High Amounts of IL-12p70 in Primary Human Monocytes. J Immunol (2009) 182:6824-33. doi: 10.4049/jimmunol.0803001

45. Coch C, Hommertgen B, Zillinger T, Daßler-Plenker J, Putschli B, Nastaly M, et al. Human TLR8 Senses RNA From Plasmodium Falciparum-Infected Red Blood Cells Which Is Uniquely Required for the IFN- $\gamma$ Response in NK Cells. Front Immunol (2019) 10:371. doi: 10.3389/fimmu.2019.00371

46. Biscans A, Coles A, Haraszti R, Echeverria D, Hassler M, Osborn M, et al. Diverse Lipid Conjugates for Functional Extra-Hepatic siRNA Delivery In Vivo. Nucleic Acids Res (2019) 47:1082-96. doi: 10.1093/nar/gky1239

47. Korolowizc KE, Li B, Huang X, Yon C, Rodrigo E, Corpuz M, et al. LiverTargeted Toll-Like Receptor 7 Agonist Combined With Entecavir Promotes a Functional Cure in the Woodchuck Model of Hepatitis B Virus. Hepatol Commun (2019) 3:1296-310. doi: 10.1002/hep4.1397

48. Lanford RE, Guerra B, Chavez D, Giavedoni L, Hodara VL, Brasky KM, et al. GS-9620, an Oral Agonist of Toll-Like Receptor-7, Induces Prolonged Suppression of Hepatitis B Virus in Chronically Infected Chimpanzees. Gastroenterology (2013) 144:1508-17, 1517.e1-10. doi: 10.1053/j.gastro. 2013.02.003

49. Niu C, Li L, Daffis S, Lucifora J, Bonnin M, Maadadi S, et al. Toll-Like Receptor 7 Agonist GS-9620 Induces Prolonged Inhibition of HBV via a Type I Interferon-Dependent Mechanism. J Hepatol (2018) 68:922-31. doi: 10.1016/j.jhep.2017.12.007

Conflict of Interest: The authors declare that the research was conducted in the absence of any commercial or financial relationships that could be construed as a potential conflict of interest.

Publisher's Note: All claims expressed in this article are solely those of the authors and do not necessarily represent those of their affiliated organizations, or those of the publisher, the editors and the reviewers. Any product that may be evaluated in this article, or claim that may be made by its manufacturer, is not guaranteed or endorsed by the publisher.

Copyright (c) 2022 Obermann, Lederbogen, Steele, Dorna, Sander, Engelhardt, Bakowsky, Kaufmann and Bauer. This is an open-access article distributed under the terms of the Creative Commons Attribution License (CC BY). The use, distribution or reproduction in other forums is permitted, provided the original author(s) and the copyright owner(s) are credited and that the original publication in this journal is cited, in accordance with accepted academic practice. No use, distribution or reproduction is permitted which does not comply with these terms. 